Journal of Animal and Veterinary Advances 11 (14): 2432-2435, 2012

ISSN: $1680-5593$

(C) Medwell Journals, 2012

\title{
Amplification of Nucleoprotein (NP) Gene from Canine Influenza Virus H3N2 and its Expression in Escherichia coli
}

\author{
${ }^{1,2}$ Wang Li, ${ }^{1,3}$ Guo Yu-Fei, ${ }^{2} \mathrm{Qi}$ Hai-Tao, ${ }^{2} \mathrm{Su}$ Shuo, ${ }^{2} \mathrm{Chen}$ Ji-Dang, ${ }^{2} \mathrm{Zhao}$ Fu-Rong, ${ }^{2} \mathrm{Cao}$ Nan, \\ ${ }^{2}$ Zhang Min-Ze, ${ }^{2}$ Zhang Chao-Yi, ${ }^{2}$ Huang Zhen, ${ }^{2}$ Tan Li-Kai, ${ }^{2} \mathrm{Li}$ Shou-Jun and ${ }^{2}$ Zhang Gui-Hong \\ ${ }^{1}$ College of Veterinary Medicine, Sichuan Agricultural University, \\ 625014 Yaan, People's Republic of China \\ ${ }^{2}$ College of Veterinary Medicine, South China Agricultural University, \\ 510642 Guangzhou, People's Republic of China \\ ${ }^{3}$ College of Animal Science and Veterinary Medicine, Jinling Institute of Technology, \\ 210038 Nanjing, China
}

\begin{abstract}
NP is a relatively conservative structure protein also is the main recognition antigen and have good middle cross reactive. To express Canine Influenza Virus (CIV) subtype H3N2 NP protein, the $N P$ gene was amplified by PCR and cloned into vector $\mathrm{pMD18-T}$ and then sequence determination. The $N P$ gene was cloned into vector pET-32a $(+)$. The recombinant plasmid $\mathrm{pET}-\mathrm{NP}$ was transformed into Rosetta II (DE3) and induced with IPTG.
\end{abstract}

Key words: Canine influenza virus, $N P$ gene, cloning, gene expression, protein

\section{INTRODUCTION}

Canine Influenza (CI) is a kind of respiratory infectious disease caused by CIV which was attributed to the virus family Orthomyxoviridae type an influenza virus. Dogs can be infected with this virus and the symptoms similar to the canine cough (Wei et al., 2011). It had reported that avian origin $\mathrm{H} 3 \mathrm{~N} 2$ subtype CIVs were isolated from dogs between May 2006 and October 2007 in South China (Jun et al., 2010) sequence alignment results showed that these isolates were similar with the H3N2 subtype isolates from canine in South Korea in 2007 (Jun et al., 2010). Influenza virus causes disastrous problems in recent years, especially for highly pathogenic avian influenza in poultry not only outcomes a destructive economic loss but also life-threatening to human beings (Du et al., 2010). Influenza virus genome contains a segmented negative, single-stranded RNA and the virus encodes at least 10 viral proteins. The basis of the $N P$ gene can be used as diagnosis of the type of a particular protein the virus and influenza virus sub-type diagnosis (Min and Ling, 2010). The phylogenetic tree of influenza A virus from different hosts show that the $N P$ genes are very conservative and the NP protein functions in phosphorylation in the viral life cycle that is still unclear (Min and Ling, 2010). Therefore, this study of CIV H3N2 subtype expression of NP is building the foundation for the influenza virus and the diagnosis.

\section{MATERIALS AND METHODS}

Influenza viruses used in this study is an $\mathrm{H} 3 \mathrm{~N} 2$ influenza virus A/Canine/Guangzhou/01/2007 (H3N2). The negative serum were Separated and stored by the laboratory. TRIzol, ExTaq, DNA polymerase, T4 DNA ligase, Reverse transcriptase, EcoRI, Xhol I and DNA Marker were all purchased from TaKaRa. Small amount of plasmid extraction kit was purchased from OMEGA. Small amount of gel kit was purchased from Biotechnology Co., Ltd. Shanghai Waston Prestained Protein Marker were purchased from Fermentas Horseradish Peroxidase (HRP) labeled rabbit anti-dog IgG was purchased from JACK company.

Virus purification: Laboratory strains kept by purified chick embryo and inoculated for 10 days old SPF chicken embryos, each embryo cysts $0.2 \mathrm{~mL}, 4$ days incubation at $37^{\circ} \mathrm{C}, 24$ were collected within $96 \mathrm{~h}$ of death and survival of chick embryo chorioallantoic solution, $0.5 \%$ were measured for chicken erythrocyte agglutination activity. With hemagglutination activity were further identified. Hemagglutination (HA) test; the conventional micro-method. HA-positive chicken embryo fluid and ND standard positive serum Hemagglutination Inhibition (HI)-test, ND-negative samples and then with the influenza virus $\mathrm{H} 1, \mathrm{H} 3, \mathrm{H} 5, \mathrm{H} 7$ and $\mathrm{H} 9$ standard

Corresponding Author: Li Shou-Jun, College of Veterinary Medicine, South China Agricultural University, 483 Wushan Road, 510642 Guangzhou, China 
positive serum $\mathrm{HI}$ test to determine its operating procedures and standards according to the literature (Song et al., 2008).

Primer design: Based on the isolated virus strains $1613 \mathrm{NP}$ and full-length sequencing of the expression vector pET-32a $(+)$ multiple cloning site of the analysis, design a pair of primers, the sequence is as follows: P1: 5'ATCCGAATTCATGGCGTCTCAAGGCA-3' P2: 5'-CCG CTCGAGTTAATT GTCATACTCCTCTGCA-3'.

Viral RNA extraction and PCR amplification of $N P$ gene: Using TRIzol reagent according to instructions for viral RNA extraction. Prepared by reverse transcription cDNA. The cDNA as a template for PCR amplification of the NP gene. PCR conditions: $95^{\circ} \mathrm{C} 5 \mathrm{~min} ; 94^{\circ} \mathrm{C} 1 \mathrm{~min}, 55^{\circ} \mathrm{C} 40 \mathrm{sec}$, $72^{\circ} \mathrm{C} 2 \mathrm{~min}, 30$ cycles; $72^{\circ} \mathrm{C} 10 \mathrm{~min}$. PCR products by agarose gel electrophoresis.

NP gene cloned: The purified PCR product into $\mathrm{PMD} 18-\mathrm{T}$ vector and transformed into $\mathrm{DH} 5 \alpha$ competent cells, resistant coating containing Amp + LB agar plate, grown overnight, positive clones were sequenced, sequence alignment analysis. Recombinant plasmid was named pMD-NP.

Construction and identification of recombinant expression plasmids: The plasmid pMD-NP was obtained according to the instructions of Plasmid Extraction kit. The NP gene was digested with the restriction enzymes EcoR I and Xho 1 the resultant fragment was subcloned into the expression vector pET-32a $(+)$ then transformed into Rosetta. The recombinant expression plasmid detected by restriction enzyme analysis and sequencing was named $\mathrm{pET}-\mathrm{NP}$.

Induced expression of recombinant plasmids and SDSPAGE: Researchers evaluated the expression of the recombinant $\mathrm{pET}-\mathrm{NP}$ protein by SDS-PAGE. The overnight cultures of Rosetta II, harboring recombinant plasmid pET-NP and pET-32a (negative control) were diluted 1:100 in fresh LB with ampicillin $100 \mu \mathrm{g} \mathrm{mL}^{-1}$ then incubated with shaking at $37^{\circ} \mathrm{C}$ until the absorbance at $600 \mathrm{~nm}$ (OD600) reached 0.6. Expression was induced with $1.0 \mathrm{mmol} \mathrm{L}{ }^{-1} \mathrm{IPTG}$ for $4 \mathrm{~h}$ at $37^{\circ} \mathrm{C}$ after the expression conditions were optimized. Researchers revealed that it was OmpW protein using Western Blot Methods.

Purification of the NP protein and Western blot analysis: NP fusion proteins were purified following the manufacturer's instructions. The positive serum of CIV
H3N2 subtype was used as first antibody and the HRP labeled rabbit anti-dog IgG was used as secondary antibody for Western-blot analysis.

\section{RUSULTS AND DISCUSSION}

Cloning and identification of the $N P$ gene: Primers designed by Kim, Ivanics, Zhang and Limn were used to amplify the $N P$ gene. An expected band in size of $1.5 \mathrm{~kb}$ detected by $1 \%$ agarose gel electrophoresis as shown in Fig. 1.

With the restriction sites of the $N P$ gene amplification: Using the designed and synthetic NP1 and NP2 as primers and CIV cDNA as a template for PCR amplification, the results by $1 \%$ agarose gel electrophoresis analysis was consistent with the expected molecular weight $1.5 \mathrm{~kb}$ as shown in Fig. 2.

Double digestion of recombinant plasmid the pMD-NP: The target gene fragment was subcloned into pMD18-T vector by $\mathrm{T}$ vector cloning identified by restriction enzymes EcoR I and Xhol I recombinant plasmid pMD-NP double restriction enzyme digestion to obtain a section of about $2.6 \mathrm{~kb}$ band and a band of about $1.5 \mathrm{~kb}$ indicating that the NP gene has been successfully built into the pMD18-T simple vector. The PCR products were sequenced and the identity was confirmed as shown in Fig. 3.

Appraisal of recombinant plasmid the pET-NP: The digested product was cloned into pET32a $(+)$ plasmid to

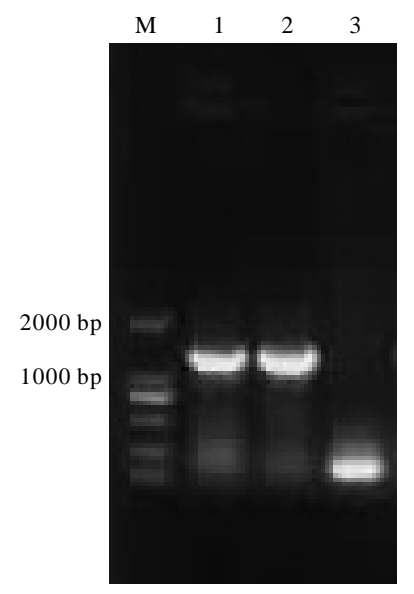

Fig. 1: Amplification of NP gene by PCR; M: DNA Marker DL2000 1: PCR products of NP gene; 2: PCR products of NP gene; $3: \mathrm{H}_{2} \mathrm{O}$ control 


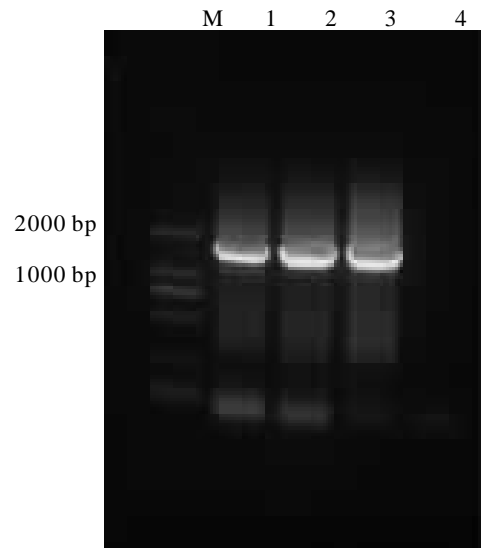

Fig. 2: Amplification of $N P$ gene by PCR; M: DNA Marker DL2000; 1: PCR products of NP gene; 2: PCR products of $N P$ gene ; 3 : PCR products of NP gene; 4: $\mathrm{H} 2 \mathrm{O}$ control

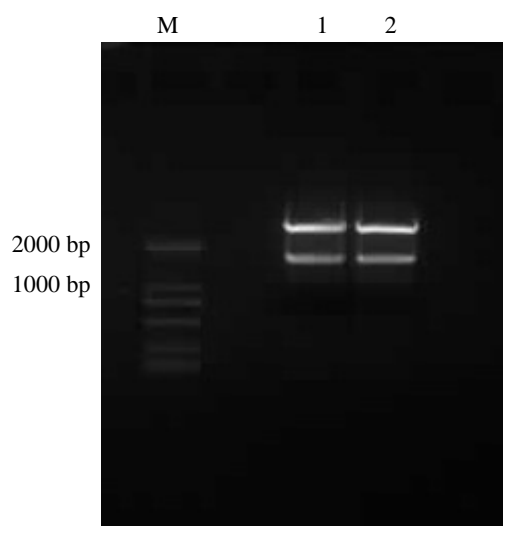

Fig. 3: Identification of recombitinant plasmid pMD-NP by enzyme digestion; M: DNA Marker DL2000 plus 1-2: pMD-NP/EcoR I+Xho I

construct pET-NP recombinant prokaryotic expression plasmid. The products of PCR were sequenced and the identity was confirmed.

Induced protein of Rosetta: The optimal induction of IPTG concentration was $1.0 \mathrm{mmol} \mathrm{L}^{-1}$. The largest amount of expression would appearance when Rosetta was induced expression for $5 \mathrm{~h}$. It was consist with the expected result that the predicted molecular mass of the NP protein was $73 \mathrm{ku}$. SDS-PAGE electrophoresis, showed that the recombinant protein existed in soluble form as shown in Fig. 4 and 5 .

Western blot analysis: Western blot analysis revealed that the NP protein has a good immune response to the

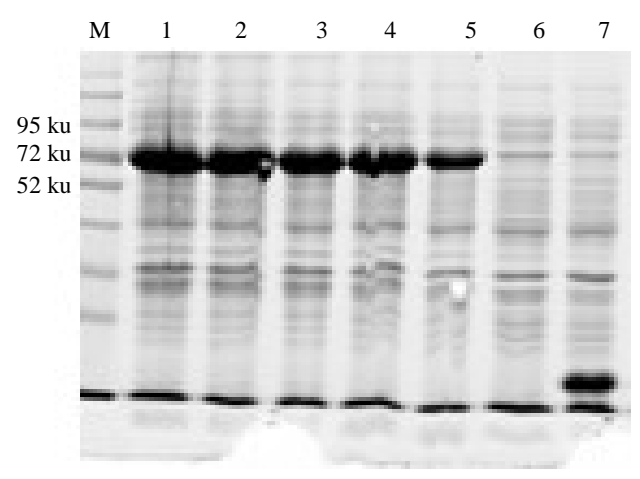

Fig. 4: Induced protein by IPTG for varied periods (1 mmol IPTG); M: Protein Marker; 7-1: Induced pET32a-NP transformed bacteria by IPTG for 0-6 $\mathrm{h}$ post-induction, respectively; 8 : Induced pET32a/Rosetta control

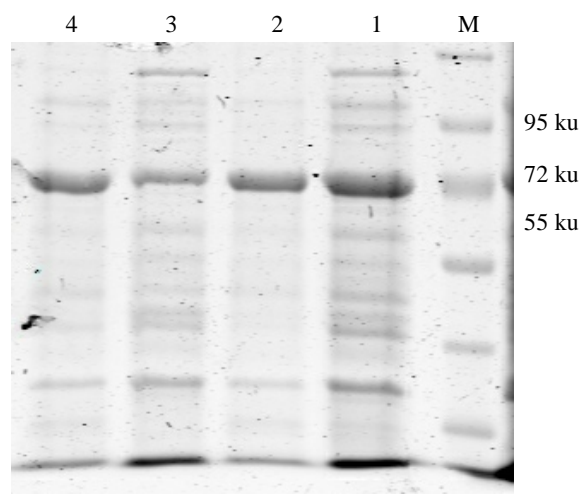

Fig. 5: Solubility analysis of the recombinant NP protein; M: Protein Marker; 1: Lysate supernatant of IPTG induced pET32a-NP/Rosetta in ultrasonic after 240 times; 2: Lysate pellet of IPTG induced pET32a-NP/Rosetta in ultrasonic after 240 times; 3: Lysate supernatant of IPTG induced pET32a-NP/Rosetta in ultrasonic after 270 times; 4: Lysate supernatant of IPTG induced pET32a-NP/Rosetta in ultrasonic after 270 times

serum as shown in Fig. 6. The pET expression system is a convenient and effective prokaryotic expression system. Target protein within the recombinant plasmid in $E$. coli can get a lot of expression by this expression system ( $\mathrm{Li}$ and Chen, 2008), so researchers chose this expression system for the $N P$ gene of influenza virus cloningand expression. Researchers successfully cloned the $N P$ gene from cloned cDNA of $\mathrm{H} 3 \mathrm{~N} 2$ canine influenza virus and constructed the prokaryotic expression system of NP protein and the protein can be better integrated and expressed in the Rosetta $\alpha$. Researchers establish the foundation for further study of its function and 


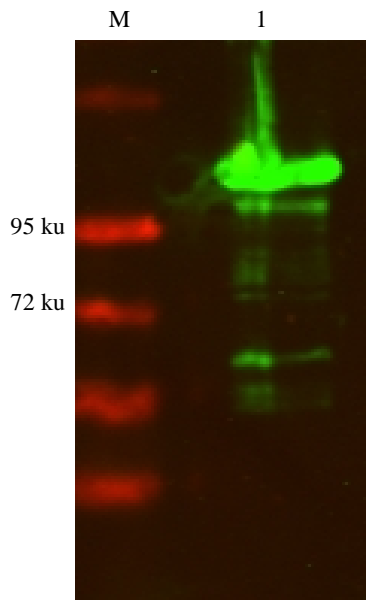

Fig. 6: Western blot analysis of the expressed NP protein M: Protein Marker; 1: pET-NP

mechanism of immune cross-protection. NP protein is influenza virus conserved structural proteins, NP and viral proteins and host cell through the interaction between certain molecules to affect viral transcription, replication, assembly and transport functions. Present mainly as a diagnostic antigen for a variety of ELISA diagnostic kit. Because of conservative nature, NP protein as a diagnostic antigen conserved influenza $\mathrm{A}$ viruses have been used for diagnostic kit development and has become a commercial product (Min and Ling, 2010; Pu et al., 2011). This study shows that experiment a large number of efficient expression of the NP protein which can further prepare for the broad-spectrum anti-NP monoclonal antibody to lay the material foundation for the different subtypes of CIV to the development of diagnostic reagents and NP protein structure and function studies provide a scientific basis and substance and material.

\section{CONCLUSION}

The expressed NP fusion protein had a MW of $73 \mathrm{ku}$ and was purified. Western blot showed that the purified NP protein could be recognized by CIV positive serum. All these results provide a basis for the preparation of efficient screening of monoclonal antibodies and diagnosis of canine influenza virus infection.

\section{REFERENCES}

Du, W.X., C.P. Jun and S. Yang, 2010. Protein expression analysis of $\mathrm{H} 3 \mathrm{~N} 2$ swine influenza virus M2. J. Biotechnol., 26: 16-21.

Jun, L.S., S.Z. Hai and J.P. Rong, 201 0. Avian-origin H3N2 canine influenza A viruses in Southern China. Infect. Genet. Evol., 10: 221-223.

Li, X.H. and H.W. Chen, 2008. Recent advance in nonstructural protein of influenza virus A. Bing Du Xue Bao., 6: 483-486.

Min, G.L. and Q.C. Ling, 2010. Protein expression and monoclonal antibody preparation of $\mathrm{H} 3 \mathrm{~N} 2$ swine influenza virus NP. Master Thesis, Huazhong University of China, China.

$\mathrm{Pu}, \mathrm{Z}$., Z. Kun and J.B. Bei, 2011. Cloning and prokaryotic expression of nonstructural protein 1 gene of a $\mathrm{h} 3 \mathrm{n} 2$ siv isolate from henan province, China. Chin. J. Biol., 24: 665-667.

Song, D., B. Kang, C. Lee, K. Jung and G. Ha et al., 2008. Transmission of avian influenza virus (H3N2) to dogs. Emerg. Infect. Dis., 14: 741-746.

Wei, Z.D., X.Y. Zhong and Y.L. Guo, 2011. Prokaryotic expression and antigenicity analysis of H3N2 canine influenza virus HAl gene. Chin. J. Virol., 2: 157-159. 